\title{
Informe del primer caso de manejo transfusional guiado por tromboelastometría rotacional en México y revisión de la bibliografía
}

\author{
Case report of patient blood managment guided by rotational thromboelastometry \\ in Mexico and literature review
}

\begin{abstract}
Ángel A. Pérez-Calatayud ${ }^{1 *}$, Luisa F. Giraldo-Cadavid, Karla Aguilar-Vidales³, Luis R. Loza-Gallardo4, Gildardo Escobar-Herrera1, Juan González-Hernández ${ }^{4}$, Socorro G. Bonilla-Casillas ${ }^{1} y$

Raúl Carrillo-Esper

${ }^{1}$ Unidad de Terapia Intensiva del Adulto, Hospital de Especialidades del Niño y la Mujer, Dr. Felipe Núñez Lara, Santiago de Querétaro, Querétaro; ${ }^{2}$ Servicio de Anestesiología, Hospital de Especialidades del Niño y la Mujer Dr. Felipe Núñez Lara, Santiago de Querétaro, Querétaro; ${ }^{3}$ Servicio de Anestesiología, Hospital de Especialidades del Niño y la Mujer Dr. Felipe Núñez Lara, Santiago de Querétaro, Querétaro; ${ }^{4}$ Unidad de Terapia Intensiva, Unidad Médica de Alta Especialidad, Instituto Mexicano del Seguro Social, Puebla, Puebla; ${ }^{5} 5$ Unidad de Terapia Intensiva, Centro Nacional de Investigación y Atención de Quemados, Instituto Nacional de Rehabilitación, Ciudad de México. México.
\end{abstract}

\begin{abstract}
Resumen
Introducción: La tromboelastometría evalúa los cambios viscoelásticos en el proceso de coagulación. Ofrece una representación gráfica de la formación del coágulo, su estabilidad y la presencia de lisis. Objetivo: Se notifica el primer caso de manejo transfusional guiado por tromboelastografía en México con revisión de la bibliografía. Método: Se realizó una búsqueda en metabuscadores (PubMed, Scielo, Medigraphic) con las palabras tromboelastometría, coagulopatía y medicina transfusional y se incluyeron los trabajos más influyentes. Conclusiones: La tromboelastometría rotacional es una herramienta diagnóstica que grafica la funcionalidad del coágulo para un manejo dirigido e individualizado de la coagulopatía relacionada con hemorragia.
\end{abstract}

PALABRAS CLAVE: Tromboelastometría rotacional. Coagulopatía. Medicina transfusional.

\begin{abstract}
Introduction: Thromboelastometry evaluates viscoelastic changes in the coagulation process. It offers a graphic representation of the formation of the coagulum, its stability and the presence of lysis. Objective: This first case of transfusion management guided by thromboelastography in Mexico and we conducted a review of the literature. Method: A metasearch search was performed (PubMed, Scielo, Medigraphic) with the words thromboelastometry, coagulopathy, transfusion medicine and the most influential works were included. Conclusions: The rotational thromboelastometry is a diagnostic tool that graphs the functionality of the clot, for a directed and individualized management of the coagulopathy associated with bleeding.
\end{abstract}

KEY WORDS: Rotational thromboelastometry. Coagulopathy. Transfusion medicine.

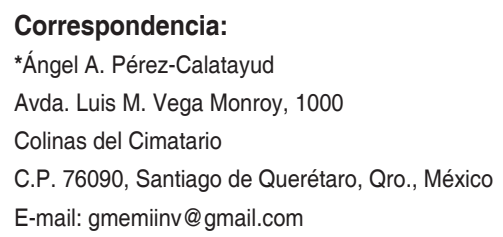

Fecha de recepción: 28-05-2018

Fecha de aceptación: 10-11-2018 DOI: $10.24875 / C I R U .18000488$
Cir Cir. 2019;87(S1):1-7

Contents available at PubMed www.cirugiaycirujanos.com 


\section{Introducción}

Hartert describió la tromboelastografía en 1948. A través de este método se evalúan los cambios viscoelásticos que experimenta la sangre durante el proceso de coagulación con la finalidad de representar de forma gráfica la polimerización de la fibrina (factores de coagulación y formación de trombina), así como el inicio de la formación del coágulo (fibrinógeno, fibrina y FXIIla) y su estabilidad (fibrinógeno y plaquetas) ${ }^{1}$, y en algunos casos la presencia de lisis del coágulo (fibrinólisis).

Se documentó el uso de la tromboelastometría con el trasplante hepático en 1985 con la finalidad de controlar la administración de hemocomponentes, y con posterioridad en la cirugía cardiaca, y hoy día se ha vuelto parte esencial del tratamiento transfusional guiado a la cabecera del enfermo de áreas críticas. El tromboelastómetro rotacional es una tecnología útil para vigilar de manera funcional la hemostasia y el tratamiento transfusional dirigido y reducir la cantidad de hemoderivados administrados y el sangrado durante los procedimientos quirúrgicos ${ }^{2}$.

El objetivo de este trabajo es notificar el primer caso de manejo transfusional guiado por tromboelastografía rotacional en México y hacer una revisión de la bibliografía.

\section{Caso clínico}

Se trata de una paciente femenina de 16 años de edad que sufrió traumatismo craneal por arma de fuego y que exigió operación de urgencia. La paciente desarrolló estado de choque hipovolémico franco, con acidosis e hipotermia que requirió grandes cantidades de vasopresores; el volumen intravascular se agotó casi por completo (pérdida sanguínea calculada de $\left.3000 \mathrm{~cm}^{3}\right)$; se transfundieron un paquete globular, plasma y cristaloides en fase de reanimación a $30 \mathrm{ml} / \mathrm{h}$. No había registros de laboratorios prequirúrgicos, a excepción de una gasometría que señaló hemoglobina de 13, con acidosis metabólica grave, $\mathrm{pH}$ de 6.8 , lactato de 13 y calcio iónico de 0.89 , sin alcanzar objetivos hemodinámicos; la hemorragia persistía, lo cual impedía que el procedimiento neuroquirúrgico concluyera la operación programada.

Al momento de correr la tromboelastometría, el EXTEM registra un tiempo de coágulo (TC) alargado de 430, con amplitud a los 5 min (A5) de 15 y una firmeza máxima del coágulo de 25 ; al realizar el
FIBTEM, el TC no se recuperó y llegó a 719 (Fig. 1); con ello se estableció la presencia de una deficiencia grave de fibrinógeno y disfunción plaquetaria.

Se indicó medicina transfusional dirigida a base de concentrado de fibrinógeno a $45 \mathrm{mg} / \mathrm{kg}$ y se inició un antifibrinolítico; con ello se logró detener la hemorragia y mejoraron los parámetros hemodinámicos, con menor uso de vasopresores, y fue posible concluir la intervención. Se efectuó también vigilancia ultrasonográfica neurológica con Doppler transcraneal de la arteria cerebral media bilateral que identificó circulación cerebral adecuada con diástole conservada (IP, 0.9; IR, 1.5) y vaina del nervio óptico de $0.4 \mathrm{~cm}$. Durante su estancia en terapia intensiva no se observó en la paciente mayor sangrado y se iniciaron medidas de paciente neurocrítico. Sin embargo, pese a la craneotomía y el control de la hipertensión intracraneal, tuvo mala evolución en terapia intensiva y sobrevino la muerte cerebral pocas horas después del postoperatorio.

\section{Fisiopatología de la coagulopatía en el traumatismo craneal}

Se prevé que en el año 2020 la lesión cerebral traumática sea la primera causa de discapacidad y muerte $^{3}$. La coagulopatía en la lesión cerebral traumática oscila entre 7 y $63 \%{ }^{4}$ y tiene una relación estrecha con la gravedad del traumatismo. Los pacientes que sufren una lesión cerebral traumática leve desarrollan coagulopatía en $<1 \%$, y en aquéllos con una lesión cerebral traumática grave o traumatismo penetrante la cifra puede ser mayor de $60 \% 5$.

Diversos mecanismos pueden explicar la coagulopatía en el paciente con una lesión cerebral traumática. Las alteraciones que se vinculan con la actividad y funcionalidad de las plaquetas se pueden dividir en dos; la primera alteración aparece cuando existe rotura de la barrera hematoencefálica, lo cual promueve la secreción del factor activador de plaquetas derivado del cerebro y el factor tisular, lo que da lugar a una hiperactividad plaquetaria y un posterior agotamiento plaquetario ${ }^{6}$. La segunda es efecto de la disfunción plaquetaria causada por una disminución de la actividad del agonista de adenosindifosfato y del ácido araquidónico debido a una inhibición de los receptores de éstos. ${ }^{7}$

Al transgredirse la barrera hematoencefálica tiene lugar una liberación masiva del factor tisular, el cual desencadena el inicio de la vía extrínseca de la coagulación al unirse al factor VIla, con la generación de trombina en la fase de inicio de la coagulación; de 

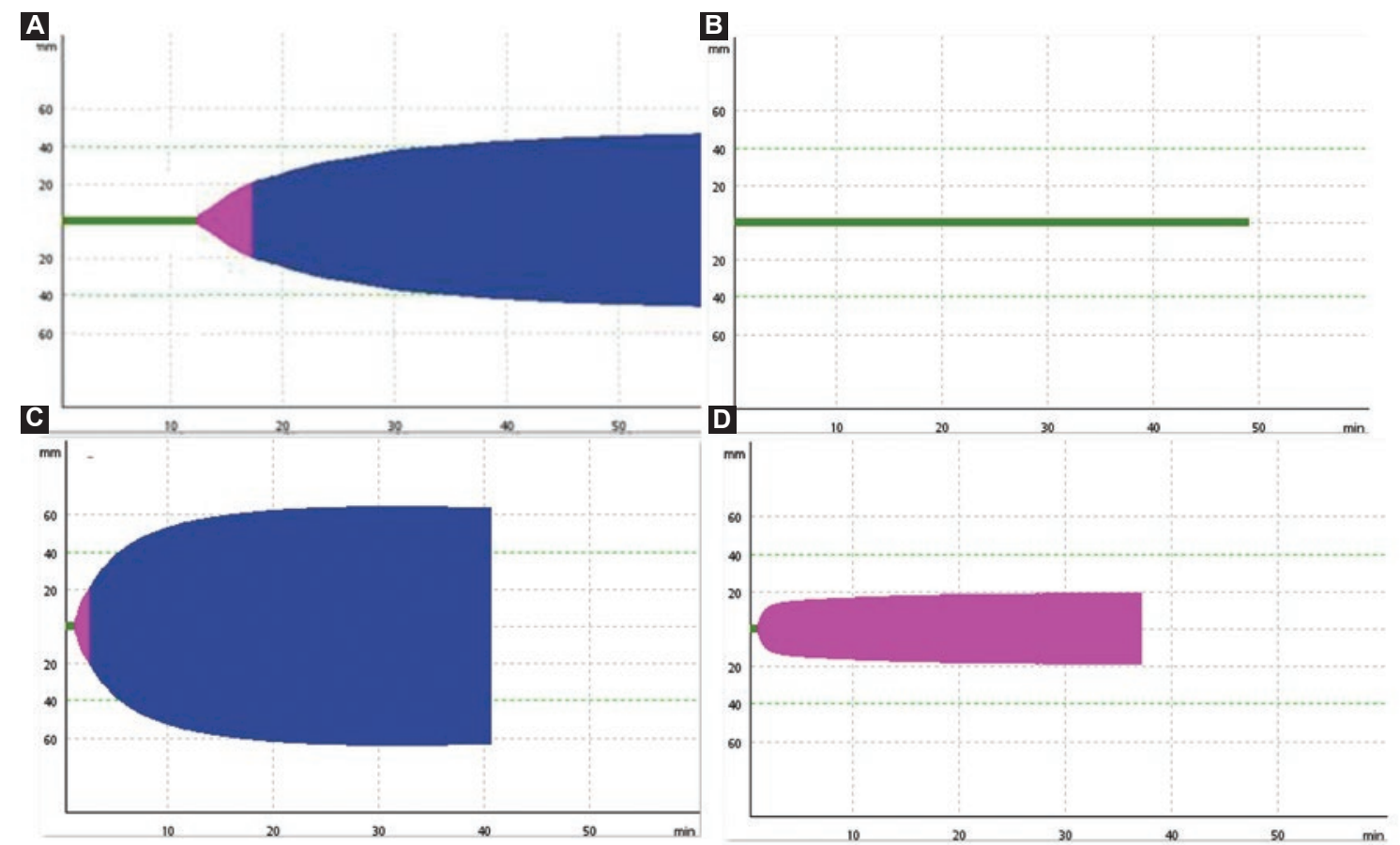

Figura 1. Tromboelastometría rotacional antes y después del manejo hemostático dirigido. A: EXTEM con un TC de $430 \mathrm{~s}, A 5$ de $9 \mathrm{~mm}$, A10 de $15 \mathrm{~mm}, \mathrm{~A} 15$ de $19 \mathrm{~mm}$, TFC de 984 s y máxima firmeza de coágulo de $25 \mathrm{~mm}$. B: FIBTEM que tras $719 \mathrm{~s}$ no presentó cambio en su amplitud. Se realizó reanimación hemostática dirigida con la administración de fibrinógeno y se corrió después de 15 min de su manejo una segunda medición con tromboelastometría. C: se observa la correspondiente a EXTEM. D: la correspondiente a FIBTEM, con una franca mejoría de la coagulopatía.

forma simultánea, dado que existen pequeñas cantidades de factor tisular soluble en la sangre, aumenta la fase de amplificación y se propicia un agotamiento de las plaquetas y el fibrinógeno ${ }^{8}$.

Los pacientes con lesión cerebral traumática secundaria a una secreción excesiva de catecolaminas pueden desarrollar un estado hiperadrenérgico; de modo adicional, el estado de hipoperfusión precipita una activación endotelial, inflamación e hiperfibrinólisis ${ }^{9}$.

La reanimación de este tipo de pacientes con administración irrestricta de cristaloides puede llevar a una coagulopatía iatrogénica al provocar hipotermia y acidosis ${ }^{10}$. Por su parte, la hipotermia inhibe la generación de trombina y la síntesis de fibrinógeno y la acidosis impide la interacción de los factores de la coagulación con los fosfolípidos de la superficie plaquetaria activada ${ }^{11}$.

\section{Principio de la tromboelastometría}

La tromboelastometría representa de manera gráfica la formación del coágulo y su lisis a la cabecera del paciente; su finalidad es evaluar la función plaquetaria, proteasas de coagulación e inhibidores de la fibrinólisis. ${ }^{12}$
La sangre se mantiene a una temperatura de $37^{\circ} \mathrm{C}$ y mediante una pipeta automática se coloca una muestra de sangre total citratada de $300 \mu$ en la cubeta (ROTEM ${ }^{\circledR}$, II Werfen México); un pin de plástico transmite la señal sobre la formación del coágulo a través de un sistema óptico, que gira hacia delante y atrás en un ángulo de $4.75^{\circ}$ y la cubeta permanece estática en este $\mathrm{caso}^{13}$. Una vez que empiezan a formarse los cordones de fibrina aumenta la fuerza de torsión entre el pin y la cubeta, lo cual se detecta ópticamente; la información generada se procesa en la computadora y aparece en forma de gráfica al final ${ }^{14}$.

\section{Módulos a medir en ROTEM}

Cada módulo de ROTEM evalúa el proceso de la coagulación; antes de agregarles el factor activador de la coagulación específico a cada una de las muestras de sangre se deben recalcificar con StarTEM que contiene $\mathrm{CaCl}_{2}$.

En el módulo EXTEM se evalúa la vía extrínseca; el factor activador de la coagulación es un factor tisular, el cual da inicio a la formación del coágulo en un periodo menor de $70 \mathrm{~s}$ y permite evaluar los 


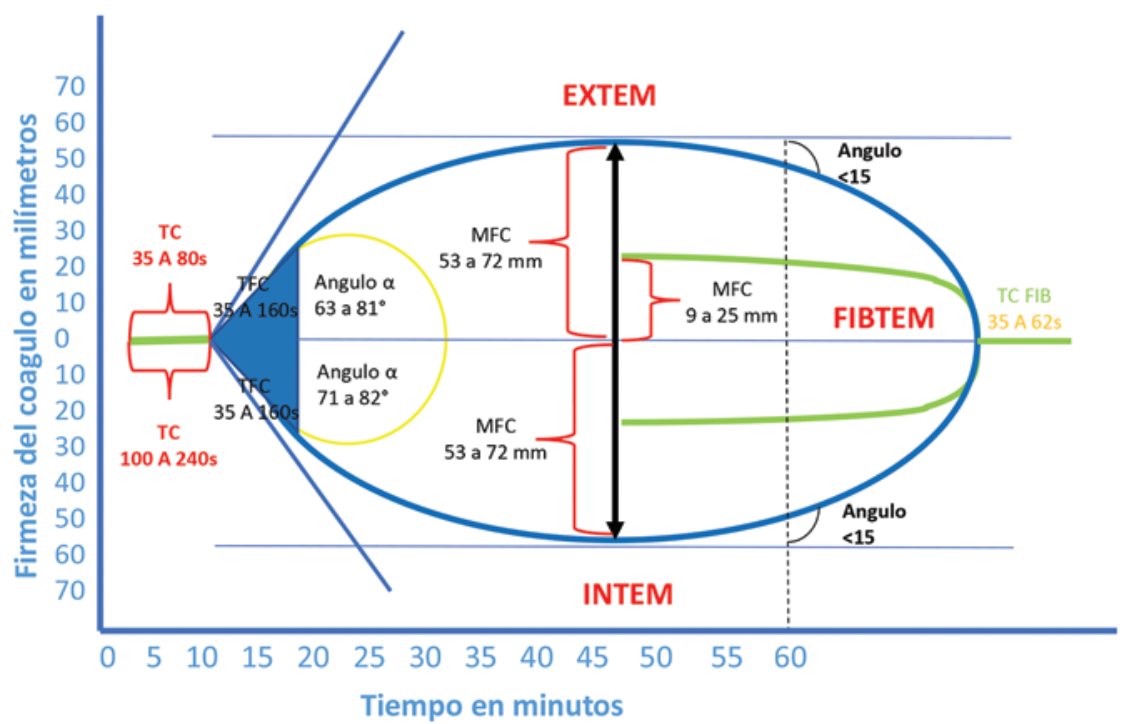

Figura 2. Gráfica de EXTEM, INTEM y FIBTEM con intervalo de valores normales. En la parte superior de la gráfica se colocan los valores normales de EXTEM, en la parte inferior de INTEM y en el lado derecho lo que corresponde a FIBTEM.

TC: tiempo de coágulo; TFC, tiempo de formación de coágulo; MFC, máxima firmeza de coágulo; EXTEM: TC de 35 a $80 \mathrm{~s}$, TFC de 35 a $160 \mathrm{~s}$, MFC de 53 a $72 \mathrm{~mm}$, ángulo $\alpha$ de 63 a 81, lisis a los $60 \mathrm{~min}$ < 15\%; INTEM: TC de 100 a 240 s, TFC de 35 a $160 \mathrm{~s}$, MFC de 53 a 72 mm, ángulo $\alpha$ de 71 a $82^{\circ}$, lisis a los $60 \mathrm{~min}<15 \%$.; FIBTEM: TC de 35 a $62 \mathrm{~s}$, MFC de 9 a $25 \mathrm{~mm}$.

factores I, II, V, VII, X de la coagulación, además de las plaquetas y la fibrinólisis ${ }^{15}$.

El módulo INTEM es un estudio activado por contacto en el que se evalúa la vía intrínseca (el factor activador es ácido elágico) y también los factores I, II, V, VIII, IX, X, XI, XII, junto con las plaquetas y la fibrinólisis ${ }^{16}$.

Al módulo FIBTEM se agrega el factor tisular más citocalacina $\mathrm{D}$, que bloquea a las plaquetas y por tanto el coágulo resultante sólo depende de la formación de fibrina y su polimerización; este módulo es el que hace posible determinar si una baja amplitud de INTEM y EXTEM se debe a la deficiencia de fibrinógeno o plaquetas ${ }^{17}$.

Al módulo APTEM se agrega factor tisular más aprotinina o ácido tranexámico con la finalidad de inhibir los procesos fibrinolíticos in vitro. Al realizar una comparación del EXTEM y el APTEN se puede identificar la fibrinólisis y precisar si el tratamiento antifibrinolítico solo es suficiente para el tratamiento 0 es necesario administrar fibrinógeno ${ }^{18}$.

Al módulo HEPTEM se añaden ácido elágico y heparinasa con la finalidad de inactivar la heparina presente en la muestra, y se determina el tiempo de coágulo en INTEM y HAPTEM; cuando el tiempo de coágulo se encuentra prolongado en la primera, pero en HAPTEN es normal, puede inferirse que existe efecto de heparina en la coagulación ${ }^{18}$.

\section{Partes que integran la curva del tromboelastograma}

La gráfica que suministra esta tecnología traza en el eje de las Y la amplitud del coágulo en milímetros y en el eje de las $X$ el tiempo transcurrido en minutos.

\section{Tiempo de coágulo (TC)}

Es el tiempo transcurrido desde el comienzo de la medición hasta el reconocimiento del inicio de la formación del coágulo y evalúa la formación de trombina y el inicio de la polimerización del coágulo ${ }^{19}$. La prolongación de este valor sugiere deficiencia de uno o más factores de coagulación; en el caso de EXTEM de la vía extrínseca y de INTEM de su prolongación, se debe por lo general al uso de heparinas ${ }^{20}$. En la gráfica puede observarse una línea plana y horizontal que culmina cuanto tiene una amplitud de $2 \mathrm{~mm}$. El intervalo promedio de normalidad del INTEM es de 100 a $240 \mathrm{~s}$, el del EXTEM de 35 a $80 \mathrm{~s}$ y el de FIBTEM de 38 a $62 \mathrm{~s}$ (Fig. 2$)^{21}$.

\section{Tiempo de formación de coágulo (TFC)}

Es el tiempo transcurrido entre el final del intervalo TC y el punto en que el coágulo adquiere una firmeza 
de $20 \mathrm{~mm}$. Los límites promedio de normalidad del INTEM son de 35 a $110 \mathrm{~s}$ y los del EXTEM de $35 \mathrm{a}$ $160 \mathrm{~s}$ (Fig. 2) 22 .

\section{Ángulo $\alpha$}

Este valor tiene estrecha relación con el TFC y se traza como una tangente en el punto de amplitud de $2 \mathrm{~mm}$ y el TFC. El intervalo promedio de normalidad del EXTEM es de 63 a $81^{\circ}$ y el del INTEM de 71 a $82^{\circ}$ (Fig. 2). Un ángulo $\alpha$ reducido y un TFC prolongado indican trombocitopenia, disfunción plaquetaria, hipofibrinogenemia o polimerización disfuncional de la fibrina ${ }^{23}$.

\section{Máxima firmeza del coágulo (MFC)}

Es la máxima amplitud de la gráfica y se mide independientemente del tiempo; esta medición representa la funcionalidad del coágulo, y al igual que en el TFC, una disminución indica trombocitopenia, disfunción plaquetaria, hipofibrinogenemia o polimerización disfuncional de la fibrina ${ }^{24}$. Es posible medir la amplitud del coágulo a los 5 o 10 min tras el inicio del TFC y con estos valores se obtiene una determinación de la MFC. MFC $=($ amplitud a los $5 \mathrm{~min}+19 \mathrm{~mm}) \pm$ $5 \mathrm{~mm} ; \mathrm{MFC}=($ amplitud a los $10 \mathrm{~min}+10 \mathrm{~mm}) \pm$ $4 \mathrm{~mm}$. El intervalo de normalidad del EXTEM es de 53 a $72 \mathrm{~mm}$, el del INTEM de 53 a $72 \mathrm{~mm}$ y el del FIBTEM de 9 a 25 mm (Fig. 2) $)^{25}$.

\section{Lisis máxima (LM)}

Señala el porcentaje de pérdida de firmeza del coágulo posterior a la MFC y puede medirse en el tiempo a los 30, 45 o $60 \mathrm{~min}$; cuando existe una pérdida de firmeza mayor al $15 \%$ a los 60 min después de MFC puede existir hiperfibrinólisis (Fig. 2) ${ }^{26}$.

\section{Uso de la tromboelastometría rotacional en traumatismos}

Todo paciente con traumatismo expresa algún grado de coagulopatía y además se expone a una reanimación excesiva con cristaloides y coloides, lo cual puede empeorar la coagulación por dilución de factores, hipotermia y acidosis; esto conduce al paciente a una hemorragia mayor, insuficiencia orgánica y, si no se corrige, a la muerte ${ }^{27}$. El inicio de la vigilancia de la coagulación se realizaba con pruebas estandarizadas de tiempo de trombina y INR; sin embargo, este tipo de estudios sólo proporciona información acerca del inicio de la formación de la fibrina, mas no de la firmeza del coágulo ni de su lisis; esto representaba una atención deficiente al vigilar a un paciente con coagulopatía y traumatismo ${ }^{28}$. Con los avances actuales, la reanimación hemostática dirigida por tromboelastometría rotacional puede hacer posible una reanimación dirigida sin exceder la cantidad de hemocomponentes, lo cual se relaciona con una mayor mortalidad y costos hospitalarios $^{29}$. En el año 2013 se condujo un estudio en el que se compararon dos cohortes de pacientes con traumatismo que requerían un protocolo de transfusión masiva; una cohorte seguía el tratamiento de hemoderivados con una relación convencional en la que se administraba por cada paquete globular plasma fresco congelado y un concentrado plaquetario; en la otra cohorte se dirigía la reanimación hemostática con pruebas viscoelásticas y se concluía que quienes recibían un tratamiento dirigido por pruebas viscoelásticas tenían una disminución de la mortalidad ${ }^{30}$. Se llevó a cabo otro estudio en el que se aleatorizó a los pacientes con traumatismo que requerían protocolo de transfusión masiva; en uno de los grupos se dirigía la reanimación con pruebas viscoelásticas y en el otro con tiempos de coagulación, fibrinógeno y plaquetas. La conclusión fue que los sujetos que eran objeto de una reanimación con medidas convencionales tenían una mayor mortalidad, así como un uso mayor de hemocomponentes ${ }^{31}$.

El uso de la tecnología de tromboelastometría rotacional no sólo tiene efecto directo sobre la mortalidad y el uso de hemocomponentes, sino que también ha permitido identificar fenotipos de coagulopatía relacionados con traumatismos. Se han descrito dos principales: la coagulopatía global con agotamiento de plaquetas y fibrinógeno y la hiperfibrinólisis. Este tipo de tecnología permite ofrecer un tratamiento hemostático individualizado ${ }^{32}$.

Contar con la tecnología de tromboelastometría rotacional y un algoritmo de manejo es de vital importancia para realizar un abordaje dirigido de la reanimación hemostática. En primera instancia es preciso identificar al paciente con traumatismo que requiera un protocolo de transfusión masiva; si el sujeto se encuentra dentro de las primeras tres horas después del traumatismo está justificado el uso de ácido tranexámico en dosis de $1 \mathrm{~g}$ por $10 \mathrm{~min}$ y luego $1 \mathrm{~g}$ en infusión por 8 h; después de este tiempo no debe administrarse de forma estandarizada, a menos que sea necesario, ya que luego de este tiempo se acompaña de un aumento de la mortalidad ${ }^{33}$. 


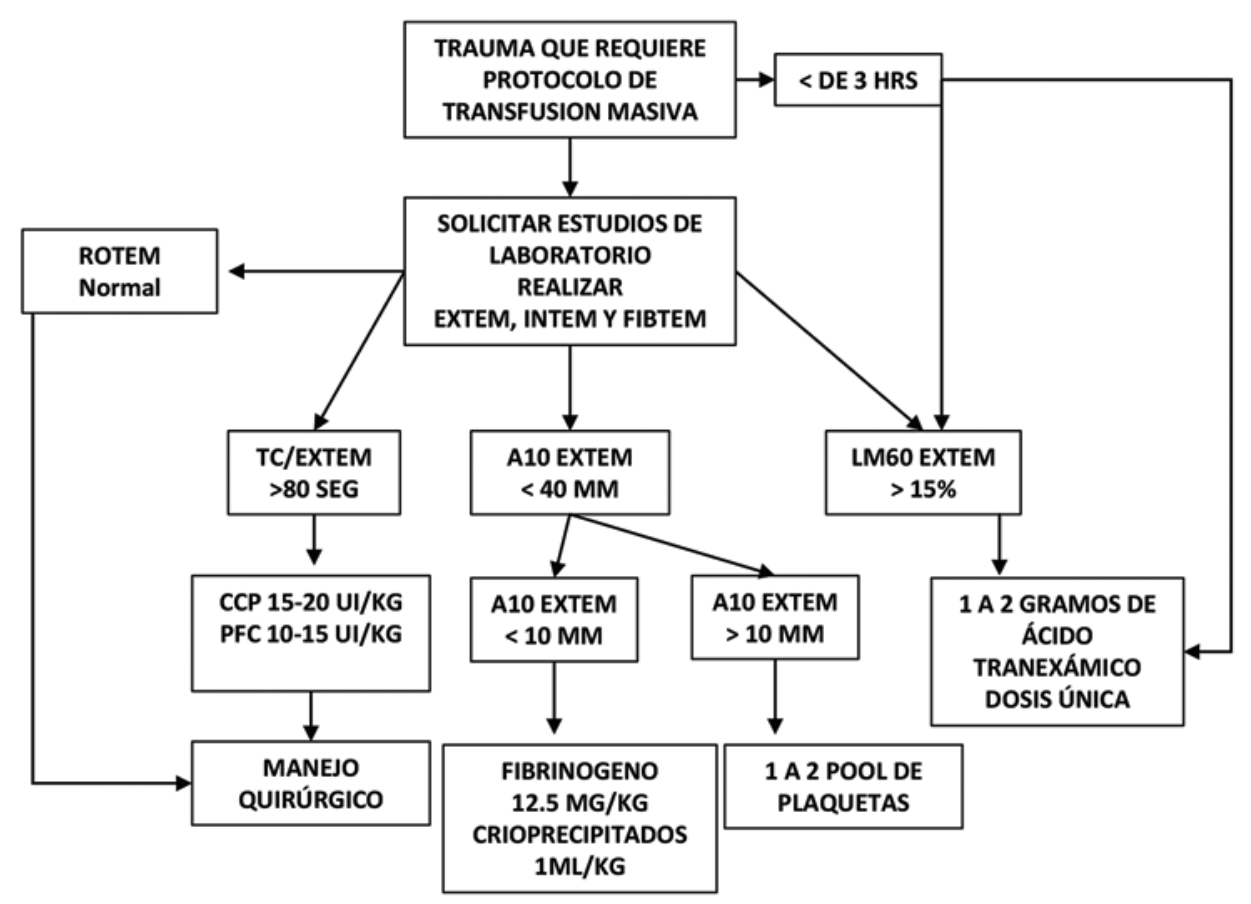

Figura 3. Algoritmo de manejo transfusional a la cabecera del enfermo con traumatismo.

TC: Tiempo de coágulo; A10: Firmeza del coágulo a los 10 min; LM60: Lisis máxima a los 60 min; CCP: Concentrado de complejo protrombínico; PFC: Plasma fresco congelado. *EI manejo con fibrinógeno y crioprecipitados depende de la amplitud en milímetros medida en FIBTEM y de cuán necesario sea llevar a éste a una amplitud de $16 \mathrm{~mm}$. Para un aumento de $2 \mathrm{~mm}$ se deben administrar $12.5 \mathrm{mg} / \mathrm{kg}$ de fibrinógeno 0 $1 \mathrm{ml} / \mathrm{kg}$ de crioprecipitados.

Se deben solicitar diversos estudios de gabinete que incluyan tiempos de coagulación, biometría hemática, fibrinógeno y pruebas viscoelásticas, en este caso tromboelastometría con EXTEM, INTEN y FIBTEM $^{34}$.

Debe efectuarse una evaluación para descartar la presencia de fibrinólisis y evaluar la firmeza del coágulo y la generación de trombina. Si el tiempo de coágulo es mayor de $80 \mathrm{~s}$ en el EXTEM debe administrarse concentrado de complejo protrombínico a una dosis de 15 a $20 \mathrm{UI}$ por kilogramo de peso o bien plasma fresco congelado a una dosis de $10 \mathrm{a} 15 \mathrm{ml} / \mathrm{kg}$. Si la lisis máxima a los 60 min es mayor de $15 \%$ se deben administrar $1 \mathrm{a} 2 \mathrm{~g}$ de ácido tranexámico y repetir sólo si es necesario.

Si la firmeza del coágulo a los 10 min en EXTEM es menor de $40 \mathrm{~mm}$ es necesario evaluar el FIBTEM y si éste es menor de $10 \mathrm{~mm}$ a los $10 \mathrm{~min}$ es preciso administrar crioprecipitados; como objetivo debe llevarse el A10 en FIBTEM a $16 \mathrm{~mm}$ y la dosis depende de cuánto sea necesario para alcanzar ese valor; por cada $2 \mathrm{~mm}$ necesarios para llevar al A10 FIBTEM a $16 \mathrm{~mm}$ se requiere 1 mililitro por kilogramo de peso de crioprecipitados o $12.5 \mathrm{mg}$ por kilogramo de peso de fibrinógeno. Por otro lado, ante un A10 en EXTEM a los
$10 \mathrm{~min}<40 \mathrm{~mm}$ con un A10 FIBTEM $>10 \mathrm{~mm}$, la disminución de la firmeza del coágulo se debe a deficiencia de plaquetas, por lo que es necesario administrar 4 a 8 unidades de plaquetas o una aféresis de éstas ${ }^{35}$.

Si después del análisis previo se identifica un trazo de tromboelastometría normal debe buscarse un origen del sangrado con medidas quirúrgicas, sin perder de vista que el objetivo es ofrecer el mejor apoyo al paciente; hay que mantener una presión arterial sistólica objetivo de 80 a $90 \mathrm{mmHg}$ siempre y cuando no exista una lesión cerebral traumática, un nivel de hemoglobina de 8 a $9 \mathrm{~g} / \mathrm{dl}$, evitar descensos de temperatura por debajo de $35.5^{\circ} \mathrm{C}$ y realizar una vigilancia del calcio ionizado (su valor normal es de 1.1 a $1.3 \mathrm{mmol} / \mathrm{L}$ ), ya que es importante para la formación y estabilización de los sitios de la polimerización de la fibrina ${ }^{36}$ (Fig. 3).

\section{Conclusiones}

La tromboelastometría rotacional es una herramienta para el manejo dirigido e individualizado de la coagulopatía que ofrece la ventaja de reducir el número de transfusiones, las complicaciones de éstas, la morbilidad y mortalidad y los costos derivados de la medicina transfusional. 


\section{Conflicto de intereses}

El autor Angel Augusto Pérez-Calatayud declara que ha participado como asesor médico y ha recibido compensación monetaria o pago de viáticos como ponente, conferencista o panelista en los últimos 36 meses por parte de Cheetah Medical México (Panamedical México), II Welfen México y Latinoamérica, LFB Pharma México, y CLS Berhing México. Asimismo es coordinador del Grupo Mexicano para el Estudio de la Medicina Intensiva (GMEMI), con el cual realiza trabajos de investigación relacionados con ultrasonografía a la cabecera del enfermo, coagulopatía en terapia intensiva, terapia intensiva obstétrica y vigilancia no invasiva.

Ha recibido apoyo por parte de la industria farmacéutica LFB y biotecnológica II Werfen y Cheetah Medical, para la realización de protocolos de investigación. Los demás autores declaran no tener conflicto de intereses.

\section{Responsabilidades éticas}

Protección de personas y animales. Los autores declaran que los procedimientos seguidos se conformaron a las normas éticas del comité de experimentación humana responsable y de acuerdo con la Asociación Médica Mundial y la Declaración de Helsinki.

Confidencialidad de los datos. Los autores declaran que han seguido los protocolos de su centro de trabajo sobre la publicación de datos de pacientes.

Derecho a la privacidad y consentimiento informado. Los autores han obtenido el consentimiento informado de los pacientes y/o sujetos referidos en el artículo. Este documento obra en poder del autor de correspondencia.

\section{Bibliografía}

1. Di Benedetto $P$, Baciarello M, Cabetti L, Martucci M, Chiaschi A, Bertini L. Thrombelastography. Present and future perspectives in clinical practice Minerva Anestesiol 2003;69:501-9.

2. Kang $Y$, Marquez D, Lewis J, Bontempo F, Shaw B, Starzl T, Winter P. Intraoperative changes in blood coagulation and thrombelastographic monitoring in liver transplantation. Anesthesia and Analgesia 1985;64:888-896.

3. Hyder A, Wunderlich CA, Puvanachandra $P$, et al. The impact of traumatic brain injuries. NeuroRehabilitation 2007;22:341-353.

4. Shehata M, Afify MI, El-Shafie M, Khaled M. Prevalence and clinical implications of coagulopathy in patients with isolated head trauma. Med J Cairo Univ 2011;79:131-137.

5. Lustenberger T, Talving P, Kobayashi L, et al. Early coagulopathy after isolated severe traumatic brain injury: relationship with hypoperfusion challenged. J Trauma 2010;69:1410-1414.

6. Deng Y, Fang W, Li Y, et al. Blood-brain barrier breakdown by PAF and protection by $X Q-1 H$ due to antagonism of PAF effects. Eur J Pharmacol 2009;616:43-47.

7. Castellino FJ, Chapman MP, Donahue DL, et al. Traumatic brain injury causes platelet adenosine diphosphate and arachidonic acid recepto inhibition independent of hemorrhagic shock in humans and rats. J Trauma Acute Care Surg 2014; 76:1169-1176.

8. Laroche M, Kutcher ME, Huang MC, Cohen MJ, Manley GT. Coagulopathy after traumatic brain injury. Neurosurgery 2012;70:1334-1345.
9. Battista AP, Rhind SG, Hutchinson MG, et al. Inflammatory cytokine and chemokine profiles are associated with patient outcome and the hyperadrenergic state following acute brain injury. J Neuroinflammation 2016;13:40.

10. Meng $\mathrm{ZH}$, Wolberg AS, Hoffman $\mathrm{M}$. The effect of temperature and $\mathrm{pH}$ on the activity of factor VIla: implications for the efficacy of high-dose factor VIla in hypothermic and acidotic patient. J Traum 2003;55:886-891.

11. Martini WZ. Coagulopathy by hypothermia and acidosis: mechanisms of thrombin generation and fibrinogen activity. J Trauma 2009;67:202-209.

12. Luddington RJ. Thrombelastography/thromboelastometry. Clin Lab Haematol 2005;27:81-90D.

13. Ganter M, Hofer C. Coagulation Monitoring: Current Techniques and Clinical Use of Viscoelastic Point-of-Care Coagulation Devices. Anesthesia \& Analgesia 2008;106:1366-1377.

14. Bolliger D, Seeberger M, Tanaka K. Principles and practice of thromboelastography in clinical coagulation management and transfusion practice. Transfus Med Rev 2012;26:1-13.

15. Weber CF, Görlinger K, Meininger D, et al. Point-of-care testing: a prospective, randomized clinical trial of efficacy in coagulopathic cardiac surgery patients. Anesthesiology 2012;117:531-547.

16. Schochl H, Cotton B, Inaba K, et al. FIBTEM provides early prediction of massive transfusion in trauma. Crit Care 2011;15:R265.

17. Song J-G, Jeong SM, Jun IG, Lee HM, Hwang GS. Five-minute parameter of thromboelastometry is sufficient to detect thrombocytopenia and hypofibrinogenaemia in patients undergoing liver transplantation. $\mathrm{Br} J$ Anaesth 2014;112:290-297.

18. Wikkelsø A, Wetterslev J, Møller AM, Afshari A. Thromboelastography (TEG) or thromboelastometry (ROTEM) to monitor haemostatic treatment versus usual care in adults or children with bleeding. Cochrane Database Syst Rev 2016;8:CD007871.

19. Jackson GN, Ashpole KJ, and Yentis SM. The TEG vs the ROTEM thromboelastography/thromboelastometry systems. Anaesthesia 2009; 64:212-215.

20. Tomori T, Hupalo D, Teranishi K. et al. Evaluation of coagulation stages of hemorrhaged swine: comparison of thromboelastography and rotational elastometry. Blood Coagul Fibrinolysis 2010;21:20-27.

21. Venema LF, Post WJ, Hendriks HG, Huet RC, et al. An assessment of clinical interchangeability of TEG and ROTEM thromboelastographic variables in cardiac surgical patients. Anesth Analg 2010;111:339-344.

22. Lang T, Bauters A, Braun SL, et al. Multi-centre investigation on reference ranges for ROTEM thromboelastometry. Blood Coagul Fibrinolysis 2005;16:301-310.

23. Hemker HC, Al Dieri R, De Smedt E, and Beguin S. Thrombin generation, a function test of the haemostatic-thrombotic system. Thromb Haemost 2006;96:553-561.

24. Afshari A, Wikkelso A, Brok J, Moller AM, and Wetterslev J. Thrombelastography (TEG) or thromboelastometry (ROTEM) to monitor haemotherapy versus usual care in patients with massive transfusion. Cochrane Database Syst Rev. 2011;3:CD007871.

25. Chitlur M, Sorensen B, Rivard GE, et al. Standardization of thromboelastography: a report from the TEG-ROTEM working group. Haemophilia 2011;17:532-537.

26. Tanaka KA, Bolliger D, Vadlamudi R, Nimmo A. Rotational thromboelastometry (ROTEM)-based coagulation management in cardiac surgery and major trauma. J Cardiothorac Vasc Anesth 2012;26:1083-1093.

27. Brohi K, et al. Acute traumatic coagulopathy. J. Trauma 2003;6:1127-1130.

28. Eckman MH, Erban JK, Singh SK, et al. Screening for the risk for bleeding or thrombosis. Ann Intern Med 2003;3:W15-W24.

29. Napolitano LM, et al. Clinical practice guideline: red blood cell transfusion in adult trauma and critical care. J. Trauma 2009;6:1439-1442.

30. Tapia NM, Chang A, Norman M, et al. TEG-guided resuscitation is superior to standardized MTP resuscitation in massively transfused penetrating trauma patients. J Trauma Acute Care Surg 2013;2:378-385.

31. González E, Moore EE, Moore HB, et al. Goal-directed hemostatic resuscitation of trauma-induced coagulopathy: a pragmatic randomized clinical trial comparing a viscoelastic assay to conventional coagulation assays. Ann Surg 2016;6:1051-1059.

32. Kutcher ME, Ferguson AR, Cohen MJ. A principal component analysis of coagulation after trauma. J Trauma Acute Care Surg 2013;5:1223-1229.

33. Shakur H, Roberts I, Bautista R, et al. Effects of tranexamic acid on death, vascular occlusive events, and blood transfusion in traumatismo patients with significant haemorrhage (CRASH-2): a randomised, placebo-controlled trial. Lancet 2010;9734:23-32.

34. Rattanabannakit $\mathrm{C}$, Nilanont $\mathrm{Y}$, Komoltri C, Prayoonwiwat $\mathrm{N}$, Poungvarin N. Accuracy and clinical utility of a portable coagulometer in an emergency setting. J Med Assoc Thai 2011;94:S89-93.

35. Gorlinger K, Fries D, Dirkmann D, Weber CF, Hanke AA, Schochl H Reduction of fresh frozen plasma requirements by perioperative point-ofcare coagulation management with early calculated goal-directed therapy. Transfus Med Hemother. 2012;39:104-113.

36. Hebert PC, Wells G, Blajchman MA, Marshall J, Martin C, Pagliarello G, et al. A multicenter, randomized, controlled clinical trial of transfusion requirements in critical care. Transfusion Requirements in Critical Care Investigators, Canadian Critical Care Trials Group. N Engl J Med 1999;340:409-417. 